\title{
Ferramenta de Prototipagem para Criação de um Aplicativo para o Ensino na Saúde
}

\author{
Karla A. Silva do Nascimento ${ }^{1}$, Lia P. Gomes Cordeiro ${ }^{1}$, Beatrice A. Duarte ${ }^{1}$, Julio \\ Cesar Couto Bem Siqueira Telles ${ }^{1}$
}

\author{
${ }^{1}$ Centro Universitário Christus (Unichristus) \\ Caixa Postal 60.192-345 - Fortaleza - CE - Brasil \\ \{karla.asn@gmail.com, liacoordeiro@gmail.com, bibsad47@gmail.com, \\ juliocctelles@gmail.com\}
}

\begin{abstract}
Social isolation has altered the routine of many educational institutions that seek, in technologies, a solution to continue the pedagogical process. However, there is a scarcity of these resources in health courses. The present work aims to analyze the application of Figma during the creation of an educational application for hematological teaching in the medical course. The study follows a qualitative, action-research approach. It was found that the resource enables interaction, information sharing and a great collaboration space.
\end{abstract}

Resumo. $O$ isolamento social alterou a rotina de muitas instituições de ensino que buscam, nas tecnologias, uma solução para dar continuidade ao processo pedagógico. Todavia, percebe-se escassez desses recursos nos cursos da área da saúde. O presente trabalho tem como objetivo analisar a aplicação do Figma durante a criação de um protótipo do aplicativo educativo "Conhecendo as Anemias" para o ensino hematológico no curso de medicina. $O$ estudo segue uma abordagem qualitativa, do tipo pesquisa-ação. Constatou-se que a ferramenta possibilita interação, compartilhamento de informações e um ótimo espaço de colaboração.

\section{Introdução}

Com o isolamento social, a fim de evitar o contágio, todas as instituições de ensino no mundo precisaram virtualizar suas aulas presenciais para dar continuidade ao processo educativo, tanto nas suas condutas pedagógicas e metodológicas quanto de gestão, [Unesco, 2020].

Dadas as circunstâncias, no Brasil o contexto não é diferente. A busca por estratégias pedagógicas rápidas e eficientes se tornou um desafio de comparações proporcionais ao combate da Covid-19. Todavia, tem-se estimulado a criação de aplicativos educativos para auxiliar o ensino remoto e as aulas com atividades síncronas e assíncronas. Apesar disso, os cursos da área da saúde possuem escassez de objetos de aprendizagem que auxiliem o ensino hematológico.

Conforme Nascimento (2019), a utilização dessas tecnologias promove práticas pedagógicas colaborativas, nas quais os estudantes também constroem conhecimentos mediante autonomia. Isso porque, possibilita que a aprendizagem aconteça em uma rede de atividades e envolvam diferentes planos sociais (indivíduos, grupos, toda a sala de 
aula), tarefas, divisão do trabalho, papéis a serem desempenhados. Assim, tanto o docente quanto o discente da saúde poderão, a partir da compreensão das especificidades da sua área de estudo, contribuir com a produção de aplicativos. Apesar disso, percebe-se que em muitos momentos o emprego dessas tecnologias em sala de aula se resume a apresentação de slides, vídeos, filmes e imagens.

Com a finalidade de contribuir para a criação de aplicativos educativos na área da saúde, o Figma (https://www.figma.com/), plataforma na qual usuários e/ou equipes projetam juntas um produto, foi utilizado para que os envolvidos em um processo criativo, pudessem dar vida ao aplicativo por meio de um protótipo de design. O Figma funciona em qualquer sistema operacional que execute um navegador da web.

Diante disso, o presente estudo tem como objetivo analisar a aplicação do Figma durante a criação de um protótipo para o aplicativo educativo "Conhecendo as Anemias" para o ensino hematológico no curso de medicina.

\section{Métodos}

O presente estudo tem uma abordagem qualitativa do tipo pesquisa-ação [Ludovico et al., 2019]. O processo de investigação se realizou no coletivo, em que o tempo e o espaço de reflexão do ato de fazer foram referendados pelos próprios participantes da pesquisa. As estratégias aqui utilizadas foram realizadas no Programa de Iniciação Científica, a partir do projeto "Aprendizagem Móvel por meio de aplicativo online para o ensino na saúde" com duas alunas do curso de medicina do Centro Universitário Christus - Unichristus.

Cada reunião foi registrada em um formulário próprio da IES. As definições sobre todas as características e funcionalidades do aplicativo e o processo de design foram elaborados por meio do Figma. A proposta foi analisar a aplicação dessa ferramenta durante a criação de um protótipo do aplicativo "Conhecendo as Anemias" para o ensino hematológico no curso de medicina. Para isso, as ideias e tomadas de decisões foram realizadas no Figma. Após cada apresentação do protótipo nas reuniões, foram coletados dados referentes ao espaço colaborativo da ferramenta $\mathrm{e}$ compartilhamento das informações em três etapas desenvolvidas: i) desenho das telas iniciais, ii) tratamento das informações sobre as anemias e, iii) distribuição dos assuntos de cada tipo de anemia, observando o seu potencial de uso.

\section{Resultados e discussão}

Sabe-se que o design é um elemento importante em um aplicativo, mas para que esse processo seja criado a contento é necessário organização e seleção das fontes certas. Dessa forma, a ferramenta Figma, objeto deste estudo, apresenta um ambiente favorável para tal criação, conforme apresentado a seguir.

O Figma faz tudo na nuvem, permite parcerias com desenvolvedores mesmo que estejam distantes geograficamente, pois possui um espaço de colaboração. Foi utilizando esse ambiente que ideias surgiram durante a prototipagem de um recurso educativo para o ensino de hematologia (ver Figura 1).

A análise da aplicação foi desenvolvida conforme a organização das telas e das suas conexões alimentadas no sistema por informações específicas sobre síndromes anêmicas, assunto mais importante do aplicativo. Além disso, percebeu-se que cada 
ação era registrada e visualizada por qualquer participante do projeto, pois cada pessoa tem um cursor nomeado para acompanhar quem está fazendo o quê. Toda vez que uma pessoa visualiza e edita um arquivo é mostrada na parte superior da ferramenta como avatares.

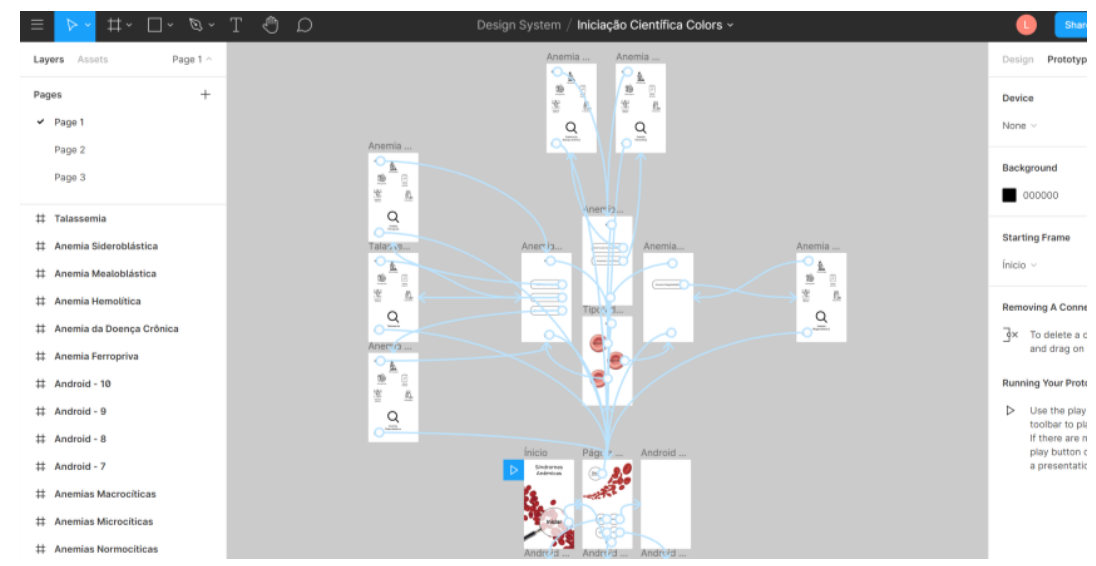

Figure 1. Elaboração do aplicativo para o ensino de Hematologia no espaço de trabalho do Figma

Diante disso, todo esse movimento promoveu a colaboração na criação e no design do produto. Segundo Braga (2014), a produção colaborativa mediante recursos ubíquos, acaba ficando em segundo plano e quase esquecido.

$\mathrm{Na}$ primeira etapa, as telas iniciais foram desenhadas. Esse trabalho foi feito de maneira simultânea, quando a equipe se reunia para realização de uma tarefa no espaço do Figma. Isso permitiu envolvimento entre as estudantes, professora e a proposta do projeto, principalmente no que diz respeito às interações produtivas, como: colaboração, argumentação, negociação e regulação mútua. Inicialmente, criou-se as telas: abertura, menu e tipos de anemias, selecionando um layout simples, leve (ver Figura 2).

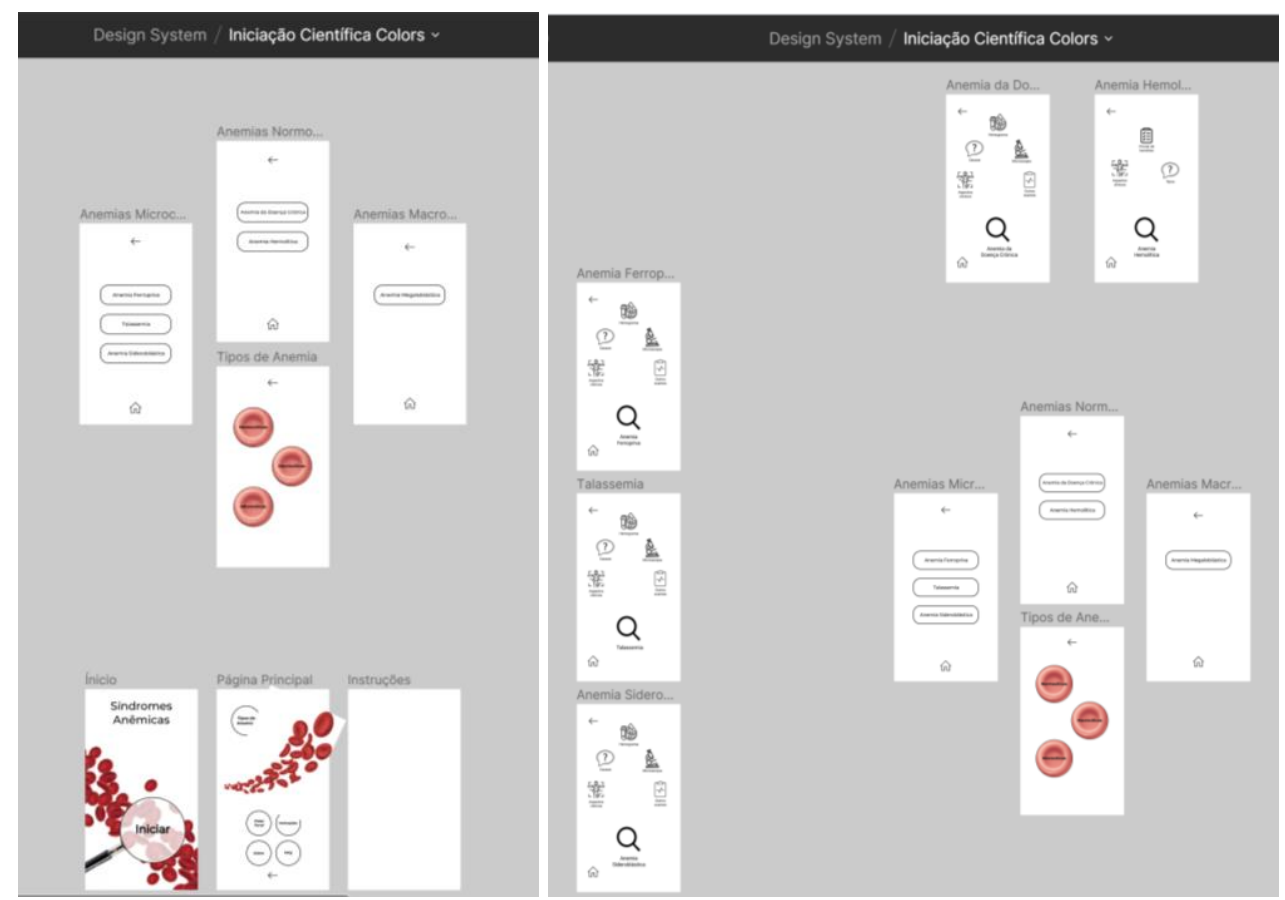

Figure 2. Estrutura com as telas principais 
A segunda etapa se deu com o desenho e tratamento das informações sobre as anemias. O Figma dispõe de uma interface amigável, assim, a equipe conseguiu definir a interação entre as telas de maneira descomplicada. Ou seja, sem necessitar conhecimento em alguma linguagem de programação. Assim, as alunas conseguiram desenvolver um protótipo com navegação entre telas e animações com botões simplificados.

Uma das características dessa ferramenta é seu potencial de envolver vários componentes, meios e funções. A equipe pode desfrutar de um elevado grau de colaboração, visto que a ferramenta cria uma rede compartilhada, socialmente interativa. Conforme Nascimento (2019), os estudantes podem produzir recursos diversos (aplicativos, vídeos, imagens etc.) a todo tempo e lugar, trocar e compartilhar informações, reforçando o imediatismo da experiência da aprendizagem móvel.

Essa ação possibilitou muito mais do que "aprender fazendo" e envolveu a equipe em uma completa participação e, consequentemente, a elaboração de significado. Em outros termos, o desenvolvimento de estratégias de ensino com essas tecnologias proporciona contextos de aprendizagens diversos [Braga, 2014].

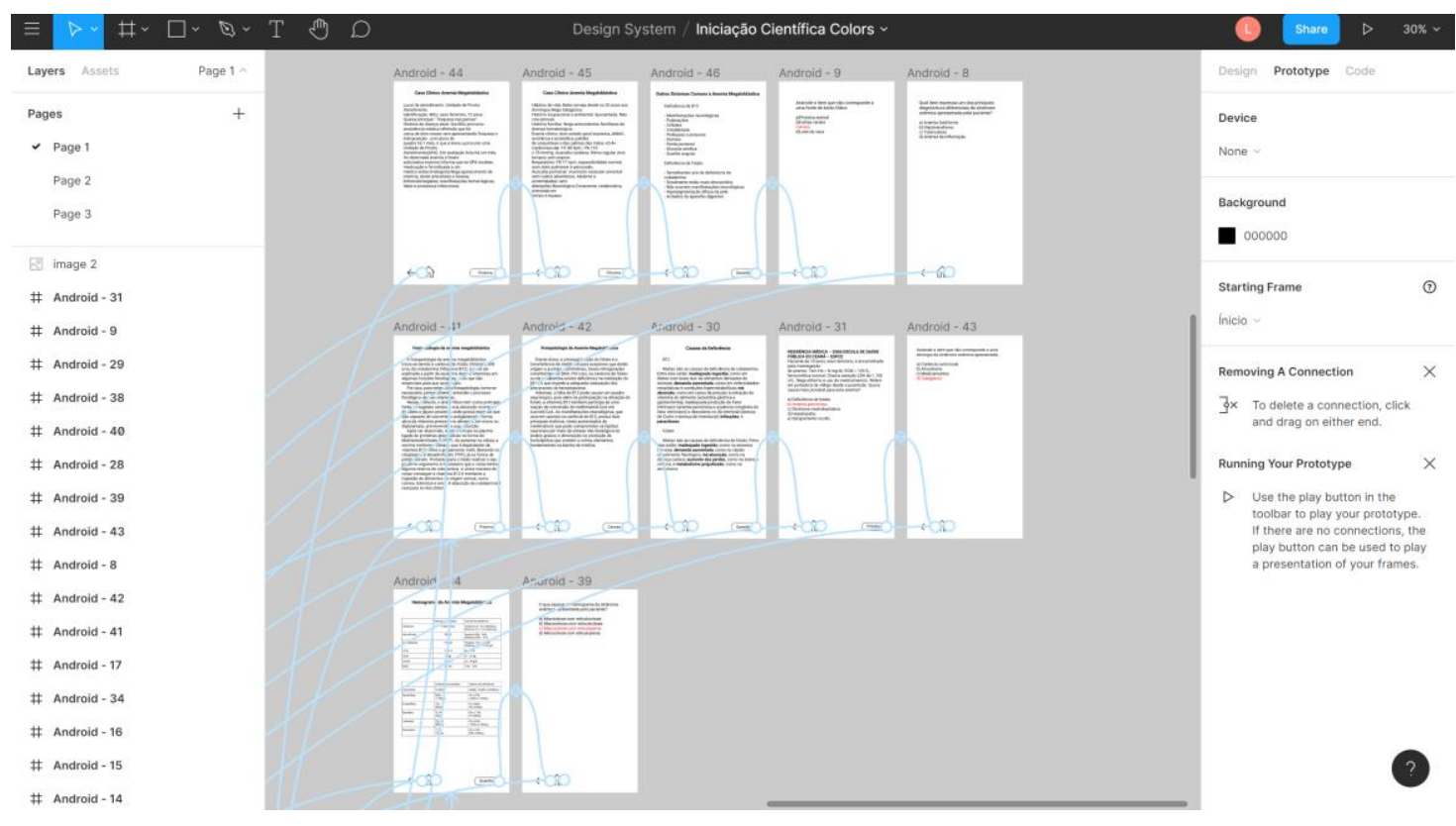

Figure 3. Telas dos recursos para o menu das atividades

Por fim, a terceira etapa possibilitou uma distribuição dos assuntos de cada tipo de anemia. Além disso, melhor organização dos botões e suas conexões, conforme o tipo de recurso específico de cada assunto, a partir de imagens, textos, links de artigos científicos, gráficos, exemplos de hemogramas, quizzes e casos clínicos.

Segundo estudos de Berribili e Mill (2018), Fonseca e Balbino (2019) e Triguero (2018), essas diferentes mídias ajudam no processo de ensino e aprendizagem, principalmente quando assumem diferentes contextos: de sala de aula, no ensino remoto, em um ambiente profissional etc. Isso porque, ainda conforme os autores, os recursos digitais possuem novos estímulos perceptivos para a produção e compreensão do conhecimento, assim, é necessário reconhecer sua importância. 


\section{Conclusões}

O estudo analisou a aplicação do Figma durante a criação de um protótipo do aplicativo educativo "Conhecendo as Anemias" para o ensino hematológico no curso de medicina. Tal recurso possui uma interface bem organizada, tornando-o fácil de usar. Com a prototipagem realizada no Figma, foi possível desenhá-lo, partindo da análise e proposta de produção de aplicativo para uso na sala de aula e fora dela. Embora já existam estudos sobre as experiências com tecnologias móveis, a maioria desses estudos não enfatiza o seu potencial como ferramenta de ensino remoto em cursos da saúde.

Todo esse trabalho colaborativo favoreceu a elaboração do referido protótipo no Figma, a contar com o entendimento das especificidades do ensino de hematologia. Além disso, contribuiu com a produção de aplicativo, viabilizando acesso aos conhecimentos científicos tanto nas ciências da saúde quando na computação.

Nesse sentido, o projeto propiciou conhecimento do potencial educacional surgido a partir da utilização de plataforma de elaboração de protótipos de aplicativos, Figma. Portanto, tal projeto possibilitará testar atividades voltadas para os aspectos de ensino, aprendizagem, acompanhamento e avaliação, corretamente resultados identificados, descritos e trabalhados em um assunto específico no curso de medicina. Esses resultados servirão de subsídios para futuras implementações do uso do Figma como ferramenta de prototipagem simples e de fácil acesso em uma visão ancorada nos avanços recentes.

\section{Referências}

Berribili, E., \& Mill, D. (2018). Impacto cognitivo do uso intensivo da internet: a autonomia dos estudos com dispositivos na adolescência. Educação \& Formação, 3(3), 177-188. doi: https://doi.org/10.25053/redufor.v3i9.862

Braga, J. (2014). Objetos de Aprendizagem Volume 1: introdução e fundamentos. Santo André: Editora da UFABC.

Fonseca, C. H. C., \& Balbino, F. (2019). Uma Aplicação Web para Produção de Textos Narrativos com Enredos Alternativos. In Anais dos Workshops do Congresso Brasileiro de Informática na Educação, v. 8, n. 1, p. 1272.

Ludovico, F., Machado, A., Weiand, A., \& Barcellos, P. C. C. (2019). Ferramentas Digitais para a Interação Assíncrona: análise de aplicações. In Anais dos Workshops do Congresso Brasileiro de Informática na Educação, v. 8, n. 1, p. 1389.

Nascimento, K. A. S. do (2019). Panorama das publicações científicas nacionais e internacionais sobre a aprendizagem móvel e a prática colaborativa. Educação \& Formação, 4(3), 207-229. doi: https://doi.org/10.25053/redufor.v4i12set/dez.3342

Triguero, I. M. (2018). Gamificación y tecnologías como recursos y estrategias innovadores para la enseñanza y aprendizaje de la historia. Educação \& Formação, 3(2), 3-16. doi: https://doi.org/10.25053/redufor.v3i8.267
Unesco. (2020). COVID-19 Impacto na
Educação. Disponível em: https://en.unesco.org/covid19/educationresponse. 